insbesondere für (uterine) Leiomyosarkome vor, auch wenn das Nebenwirkungsniveau kritisch zu betrachten ist.

_ Gemcitabin plus DTIC bietet bei refraktären Patienten ebenfalls eine Behandlungsalternative.

_ Gleiches gilt für Taxane beim Subtyp der vaskulären Sarkome.

Die übrigen, moderat aktiven Substanzen erzielen mediane PFS von ca. zwei bis drei Monaten bei deutlich stärker vorbehandelten Populationen. Aufgrund der heterogenen Biologie der Entitäten beginnt sich die Therapie zu diversifizieren. Beispiele hierfür sind (auszugsweise) Aromatase-In- hibitoren bei (low grade) endometrialen Stromasarkomen, Topoisomerase-Inhibitoren in Kombination bei Rhabdomyosarkomen, Imatinib/Nilotinib bei pigmentierter villonodulärer Synovialitis (PVNS), VEGFRTKIs plus Temozolomid bei solitärem fibrösem Tumor (SFT)/Hämangioperizytoma, VEGFR-Inhibitoren bei alveolärem Weichteilsarkom etc.

Pazopanib stellt in diesem Kontext eine weitere neue Behandlungsoption dar, besonders bei den Subtypen Synovial- und Leiomyosarkom. Man kann spekulieren, wie ein direkter Vergleich mit einer zugelassenen Substanz ausgefallen wäre. Die Erhe- bung der Lebensqualität verdeutlicht, dass durch den Pazopanib-induzierten Progressionsarrest das Wohlbefinden der Patienten unter einer Palliativbehandlung nicht verbessert werden konnte. Pazopanib wurde von der FDA und der EMA aufgrund der PALETTE-Daten kürzlich zugelassen.

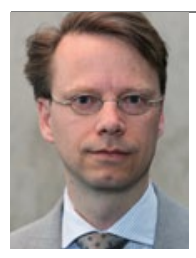

Prof. Dr. Jörg T. Hartmann Christian Albrecht Universität zu Kiel joerg.hartmann@email. uni-kiel.de

\title{
Aggressive Therapie überzeugt langfristig
}

Lokal lassen sich ausgedehnte Weichteilsarkome der Extremitäten recht gut kontrollieren. Wer aber Fernmetastasen kontrollieren und das Gesamtüberleben verlängern will, muss offenbar aggressive Therapien einsetzen.

$$
\text { L }
$$

okale Kontrolle lässt sich für Patienten mit ausgedehnten Weichteilsarkomen an den Extremitäten recht zuverlässig erreichen, doch das Risiko für Fernrezidive und frühen Tod ist signifikant. Am Massachusetts General Hospital in Boston gehen Kollegen deshalb aggressiv und multimodal gegen die Krankheit vor. Sie geben präoperative eine Chemotherapie aus Mesna, Doxorubicin, Ifosfamid und Dacarbazin (MAID) , verflechten diese mit einer Radiotherapie, entfernen dann den Tumor und fügen noch eine postoperative Chemo- oder Chemoradiotherapie an. So konnten sie Fernkontrolle und Gesamtüberleben erheblich verbessern. Aber gilt dies auch im Langzeit-Follow-up?

Die Wissenschaftler berichten nun 7-Jahres-Daten für 48 Patienten, die sie mit einer historischen, aber gematchten Population von Kontroll-Patienten vergleichen. Die erwachsenen Patienten mit einem mindestens $8 \mathrm{~cm}$ großen aggressiven Weichteilsarkom an den Extremitäten erhielten drei Zyklen MAID präoperativ sowie eine Radiotherapie mit insgesamt $44 \mathrm{~Gy}$, gefolgt von der Operation und drei Zyklen MAID postoperativ. Patienten mit positiven Operations-

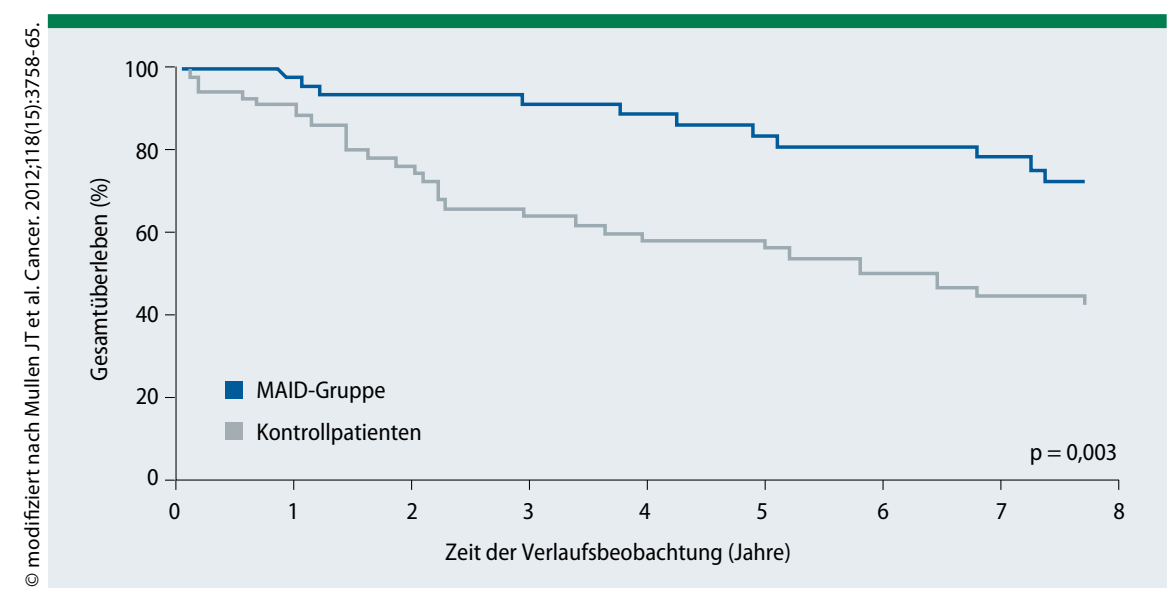

Abb. 1: Gesamtüberleben von Patienten, die Mesna, Adriamycin (Doxorubicin), Ifosfamid und Dacarbazin (MAID) erhielten und Kontrollprobanden. rändern erhielten darüber hinaus postoperativ eine Radiotherapie mit 16 Gy.

Die Behandlung wurde von 1989 bis 1999 durchgeführt. Nach median 9,3 Jahren bei den Überlebenden der MAID-Gruppe und 13,2 Jahren bei den Überlebenden der Kontrollgruppe betrug das 7-Jahres-Gesamtüberleben für die MAID-Patienten $79 \%$ und das krankheitsspezifische Überleben $81 \%$; für die Kontrollpatienten lag das Gesamtüberleben bei $50 \%$ und das krankheitsspezifische Überleben bei $45 \%$. Das extrapolierte 10-Jahres-Gesamtüberleben liegt nach MAID bei $66 \%$ und in der Kontrollpopulation bei $38 \%$.

Fünf von elf Patienten in der MAIDGruppe und sieben von 25 Kontrollpatienten starben an einem Sarkom mehr als fünf Jahre nach der Behandlung. Ein MAID-Patient entwickelte nach $53 \mathrm{Mo}-$ naten eine tödliche Myelodysplasie.

Fazit: Für Patienten mit fortgeschrittenem Weichteilsarkom der Extremitäten erreicht ein intensives Regime aus neoadjuvanter Chemoradiotherapie und Operation einen signifikanten Überlebensvorteil, der auch beim LangzeitFollow-up noch andauert. Die Autoren räumen ein, dass die kleine Probandenzahl keine allzu weitreichenden Schlüsse erlaubt: „Aber wir sind geradezu enthusiastisch über diese Langzeitergebnisse."

Christina Berndt

Mullen JT et al. Long-term follow-up of patients treated with neoadjuvant chemotherapy and radiotherapy for large, extremity soft tissue sarcomas. Cancer. 2012;118(15):3578-65. 